\title{
Noise Performance of Frequency- and Phase-Locked CW Magnetrons Operated as Current-Controlled Oscillators
}

\author{
Imran Tahir, Amos Dexter, and Richard Carter, Senior Member, IEEE
}

\begin{abstract}
Low-power continuous wave "cooker" magnetrons driven from industrial-quality switch-mode power supplies have been frequency locked by driving them as current-controlled oscillators in a phase-lock loop (PLL). The noise performance of these frequency-locked oscillators is reported as a function of heater power.

The injection of -30 - to -40 -dB signals derived from the reference oscillator of the PLL into the magnetron's output waveguide while the anode current is controlled by the PLL is shown to phase lock the magnetron's output. Results for locking performance are presented.
\end{abstract}

Index Terms-Magnetrons, noise, phase-locked loops (PLLs).

\section{INTRODUCTION}

B EFORE the introduction of the carburized, thoriated, tungsten cathodes, continuous wave (CW) magnetrons and in particular cooker magnetrons had particularly noisy spectra [1]. The poor noise performance was partly attributable to ion emission from the cathode [2]. Work sponsored by the National Aeronautics and Space Administration (NASA) and the Department of Energy (DOE) in the late 1970s revealed that magnetrons with carburized, thoriated, tungsten cathodes had very low noise levels when operated from well-filtered dc power supplies and with the cathode heater current turned off [3]. The interest in this case was to beam electrical power to earth from a solar satellite farm. Achieving this necessitated phasing the output of a large number of efficient, low weight, microwave generators.

The phase locking of oscillators and in this case magnetrons is important whenever one wishes to combine the output of a number of devices. This is of particular interest to industrial processing where it is not possible to economically build and operate $2.45-\mathrm{GHz}$ magnetrons with outputs greater than a few tens of kilowatts.

The noise performance of high-power oscillators is a key factor relating to how easily they are phase-locked and how unwanted emissions interfere with communications equipment and surrounding electronics.

It is well known that the phase and, hence, the frequency of an oscillator and indeed a magnetron can be locked by an injection

Manuscript received May 10, 2005; revised June 24, 2005. This work was supported in part by the Particle Physics and Astronomy Research Council, U.K., and in part by E2V Technologies Ltd. The review of this paper was arranged by Editor W. L. Menninger.

The authors are with the Department of Electrical Engineering, Lancaster University, Lancaster LA1 4YR, U.K. (e-mail: a.dexter@lancaster.ac.uk).

Digital Object Identifier 10.1109/TED.2005.854276 signal [4]-[7]. The injection power required to achieve locking increases with the square of the frequency difference between the injection signal and the natural frequency of the oscillator. Temperature changes and power supply ripple make the bandwidth of a free running magnetron too large for locking with an injection signal to be economic as compared to using an amplifier to generate a phase-locked signal.

If a magnetron is to be phase locked with a very low-power injection signal it is necessary to control its natural frequency of operation. Nominally, there are four ways by which the natural frequency of a magnetron can be varied and tuned. The first is with tuning stubs in the anode cavities, the second is by varying the reflected power back to the magnetron (pulling), the third is by varying the current through the magnetron (pushing) and the fourth is by varying the magnetic field.

Of these tuning methods, the most rapid is by exploiting the pushing effect. In this paper the pushing characteristic is utilized in a rapid control loop to lock the frequency. The pulling characteristic is then used to manually tune the magnetron to the required power output. With the frequency constrained to a small bandwidth, a small reference signal injected into the output waveguide can then be used to lock the magnetron phase.

\section{MAgnetron Pushing Characteristic}

When the dc voltage applied to a magnetron is increased from zero and before the magnetron starts to deliver power, a subsynchronous charge layer develops around the cathode [8]. When a pi-mode electromagnetic oscillation starts to build in the slow wave structure, waves develop on this charged layer. The crests of the waves become synchronous with the electromagnetic wave so that the crests are adjacent to the positively charged vanes at phases when the fields across the gaps between the vanes are at a maximum. As the height of the waves increases, the effective capacitance between the anode and the cathode increases and the natural frequency of the magnetron decreases [9].

As the waves grow they develop into the familiar magnetron spokes. When they reach the anode, the current path between cathode and anode is complete. Current must of course flow between the cathode and the anode for the tube to deliver power. At the operating point when the magnetron starts to deliver power and at the phase (instant) of maximum field across the gaps, the spokes are adjacent to the positively charged vanes. At this position the RF field is perpendicular to the orbital velocity of the electrons and hence very little power is transferred to the RF 
field. From this operating point, as the cathode voltage is made more negative, the spokes accelerate as a consequence of the crossed static magnetic field. The spokes move to an advanced position, i.e., at the phase of maximum field across the gaps, the spokes are advanced with respect to the positively charged vanes (and closer to the negatively charged vanes). In this position the electrons in the spokes see a de-accelerating circumferential RF field and hence deliver power to the RF field.

The crossed RF field with the static magnetic field causes the electrons to drift closer to the anode as required by energy conservation. As the power output from the tube increases, the spokes move further in advance of the positively charged vanes at the phase of maximum retarding field. This movement away from the vanes decreases the effective capacitance between the anode and the cathode. The effect of this is that the frequency of the magnetron output increases. This is the pushing effect. It should be noted that the frequency of the RF output from the tube depends on electric oscillations of the loaded slow wave structure and not the angular speed of the charged spokes.

There is a small time delay between the application of the increased voltage and the spokes settling to their new position. The frequency of the loaded slow wave structure is tied to the position of spokes. The current through the spoke is determined by its position and the RF field. Consequently the frequency is instantaneously related to the current rather than the applied voltage.

The frequency of the slow wave structure also has a strong dependence on its temperature. As the current increases the anode block collects more energetic electrons, it gains temperature and expands. For many CW magnetrons the frequency shift associated with temperature variation is of the same order of magnitude as the change due to the pushing effect. For a dynamical analysis of the magnetron it is a reasonable approximation to write

$$
\nu=f(I, T)
$$

where $\nu$ is the instantaneous frequency, $I$ is the instantaneous current in the external circuit, $T$ is the anode block temperature and $f$ gives the functional dependence. A more precise analysis would include separate temperatures for the anode block and the anode vanes.

Normally pushing curves are given without reference to the temperature hence different results are obtained if different cooling regimes are used. The frequency control loop for this work needs to act faster than thermal changes hence it is the constant temperature pushing curves that are relevant here.

Once a magnetron has been frequency locked it is straightforward to record current against temperature for constant frequency. It is then possible to construct pushing curves for constant temperature. When a magnetron is operated at constant current rather than being frequency locked, the frequency span is so large that it can be difficult to accurately measure the frequency shift as the magnetron anode heats up. Fig. 1 gives pushing curves for the Panasonic Magnetron 2M137 $1.2 \mathrm{~kW}$ driven from a dc supply with the heater power optimized for low noise. Here, the temperature has been measured on the outer

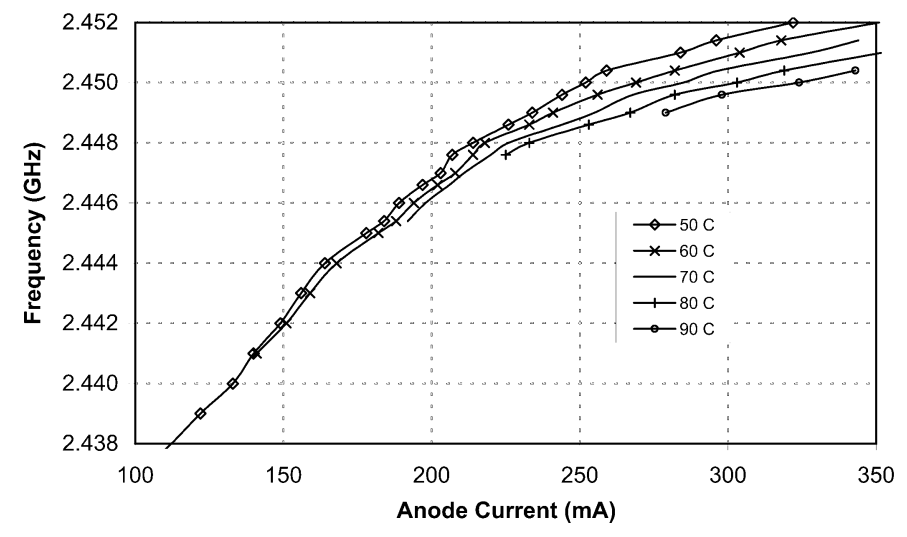

Fig. 1. Constant temperature frequency pushing curves.

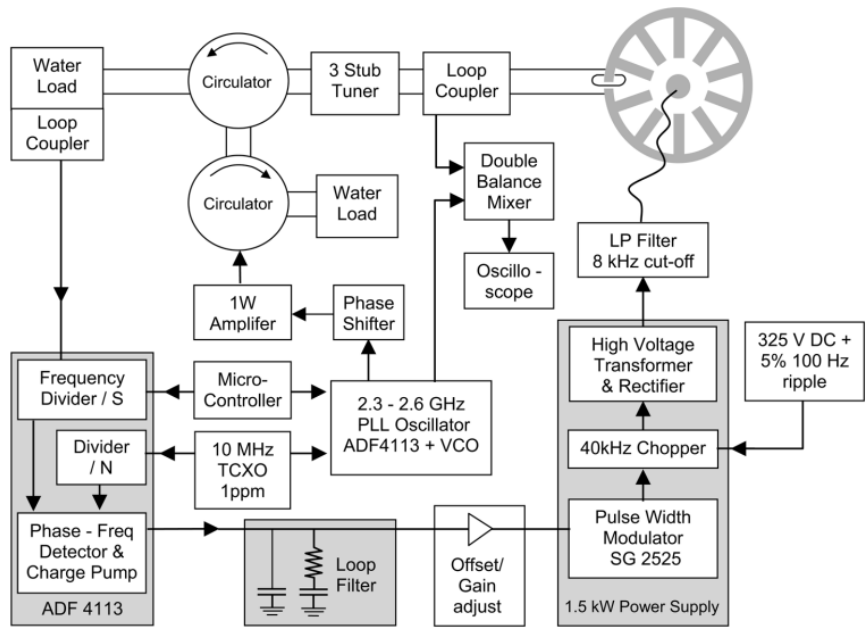

Fig. 2. Experimental arrangement for magnetron locking.

body of the anode. At $300 \mathrm{~mA}$, the variation in frequency between $50^{\circ} \mathrm{C}$ and $90^{\circ} \mathrm{C}$ is about $2 \mathrm{MHz}$. The expected variation of frequency associated with thermal expansion of the anode is given approximately as the expansivity of the copper anode, times the temperature rise, times the frequency which gives 1.6 $\mathrm{MHz}$ for a temperature rise from $50{ }^{\circ} \mathrm{C}$ to $90^{\circ} \mathrm{C}$.

\section{PLL CIRCUIT}

Phase locking has been demonstrated using Panasonic and National Magnetrons 2M137, 2M107, NL10254. Frequency control and phase locking was achieved through the control loop sketched in Fig. 2 To study PLL frequency control independently of injection locking, the injection amplifier is turned off.

The challenge for frequency and phase locking is to achieve and maintain locking for indefinite periods of time in the presence of power supply fluctuations and thermal drift. To demonstrate this the chosen power source was a switched mode power supply with minimal filtering of the dc input. Standard operation for a switched mode power supply would be to vary the pulsewidth so as to maintain either constant current or constant power. In our implementation, the pulsewidth is varied to control for constant frequency. 


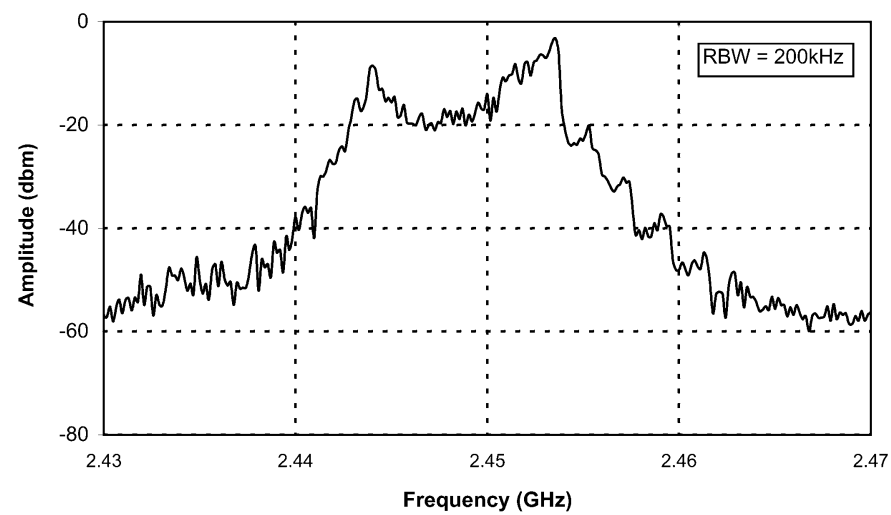

Fig. 3. Magnetron spectrum without PLL control and with full heater power $(68 \mathrm{~W})$.

Phase frequency detection was implemented with a low-cost ADF4113 ic. The output of this chip is a series of current pulses whose length is proportional to the phase difference. The measured phase difference can be up to $2 \pi S$ where $S$ is the division ratio of the input signal. The division ratio $N$ for the $10 \mathrm{MHz}$ reference signal was set as 50 for most of the work thereby permitting frequency adjustments in steps of $200 \mathrm{kHz}$. The division ratio $S$ is then determined as the required magnetron frequency divided by $200 \mathrm{kHz}$.

The loop filter acts as a proportional/integral controller and hence determines how the switch mode power supply's output varies in response to a phase error. An offset was added to the output of the loop filter so that on power up and before the phase detector receives an input, the switched mode power supply generates a small anode current sufficient for the magnetron to start (70-80 mA at 4300-3900 V dependent on filament heater power).

The absolute reference was provided by a $1 \mathrm{ppm}, 10 \mathrm{MHz}$ temperature compensated, crystal oscillator. The chosen frequency of operation is programmed by the microcontroller that sets division ratios for the frequency dividers in the two ADF4113 ics. A double-balanced mixer is used to give independent phase measurements at the microwave frequency.

Controllers used were first-order and second-order type II passive filters. Their transfer functions were optimized by standard techniques [10]. The dc input for the switched mode power supply was derived from rectified mains with minimal filtering of the ripple as would be appropriate for a low cost industrial supply. Effectiveness of the PLL can be demonstrated by its ability to vary the pulsewidth of the chopper on the switched mode power supply to maintain a relatively constant output current and voltage with evidence of the $100-\mathrm{Hz}$ ripple greatly reduced. Small modulations of the current and voltage give the precise frequency control.

\section{NOISE PERFormAnCE}

Fig. 3 shows the output spectrum of the 2M137 magnetron at a power level of $1 \mathrm{~kW}$ and with a relatively high heater power as might be set for a typical heating application without PLL frequency control or injection locking. The power supply was operated with constant pulsewidth on the modulator rather than constant current hence the spectrum includes effects associated with mains ripple. The spectrum is much broader than one would predict from the pushing curves together with the observed $+/-5 \%$ anode current ripple.

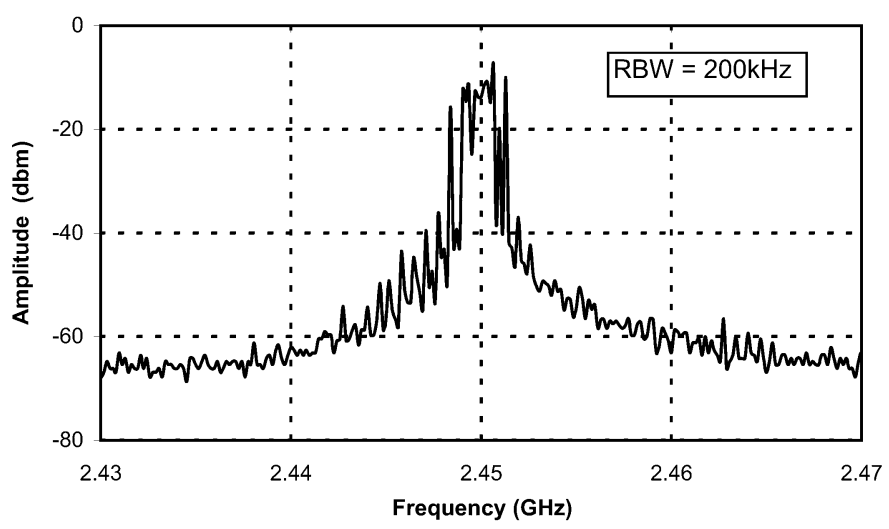

Fig. 4. Magnetron spectrum without PLL control and with $34 \mathrm{~W}$ heater power.

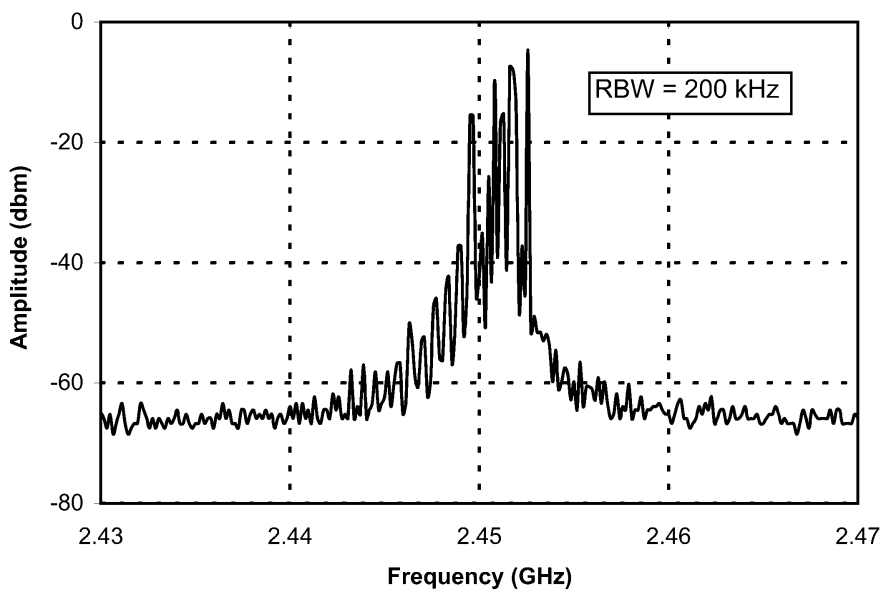

Fig. 5. Magnetron spectrum without PLL control and with 17-W heater power.

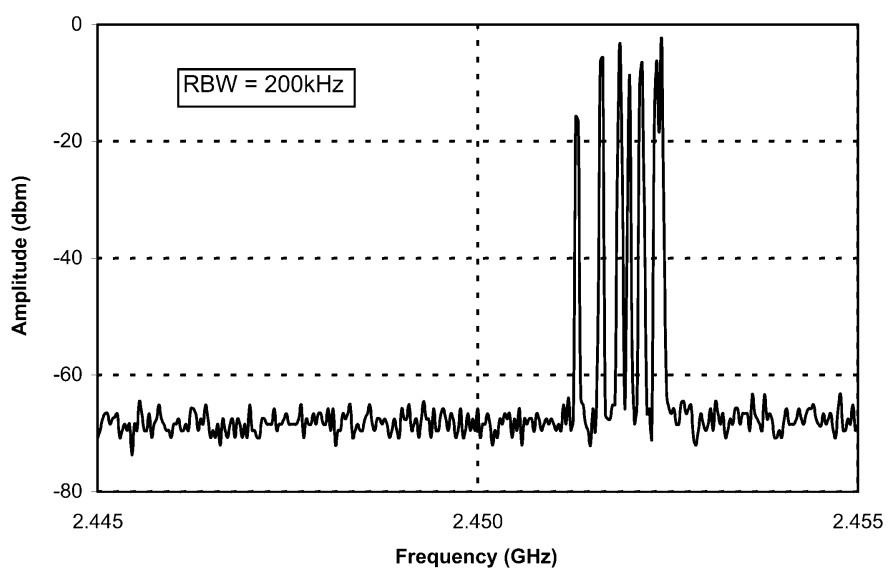

Fig. 6. Magnetron spectrum without PLL control and with $8.5 \mathrm{~W}$ heater power.

The noise performance depends strongly on the heater power relative to the power output. As the heater power is reduced the broad band spectrum narrows until at low heater powers the spectrum is composed of a number of well defined bands. This is illustrated in Figs. 4-6.

In the figures, RBW stands for the resolution bandwidth of the spectrum analyzer. This was set to $200 \mathrm{kHz}$ for Figs. 3-5 and $20 \mathrm{kHz}$ for Fig. 6.

Fig. 6 suggests that the magnetron jumps between discrete frequencies following the natural frequency that shifts with the $100 \mathrm{~Hz}$ ripple. Individual spectral peaks have bandwidths of the order of or less than $50-100 \mathrm{kHz}$. Were the magnetron to be run 


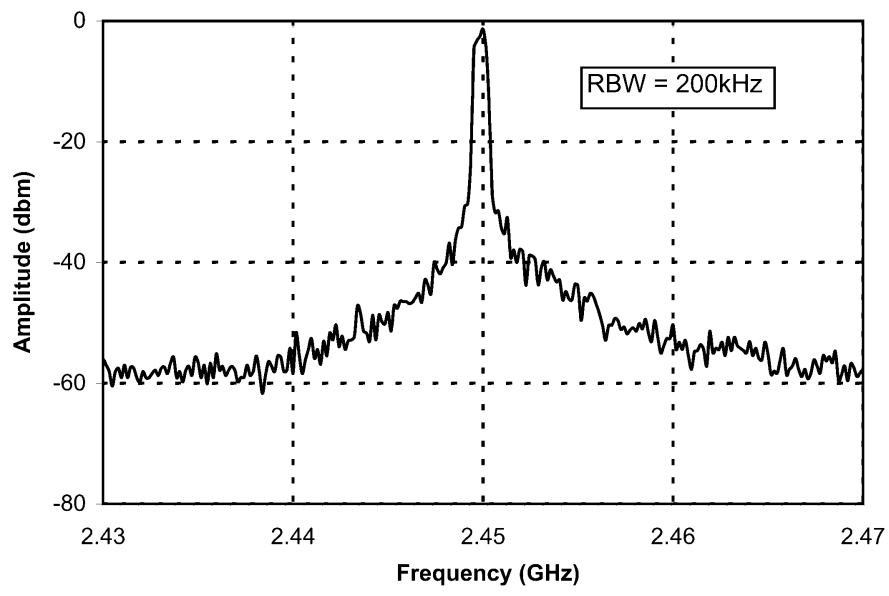

Fig. 7. Magnetron spectrum with PLL frequency control and with $34 \mathrm{~W}$ heater power.

at this operating point from a constant current power supply with zero ripple and after the magnetron's temperature has stabilized, one would see a single peak.

For the magnetron operating with $68 \mathrm{~W}$ of heater power the anode voltage was $3900 \mathrm{~V}$, the anode current $0.293 \mathrm{~mA}$ and the efficiency (RF power out divided by dc power plus heater power in was $62 \%$ ). For the magnetron operating with $8.5 \mathrm{~W}$ of heater power the anode voltage was $4080 \mathrm{~V}$, the anode current $0.289 \mathrm{~mA}$ and the efficiency (RF power out divided by dc power plus heater power in was $60 \%$ ).

In order to achieve PLL frequency control, it is necessary to reduce the noise and frequency spread below the levels associated with Fig. 3. For the 2M137 magnetron, locking becomes possible as the heater power drops to about 40 Watts, which is the heater level associated with Fig. 4. Fig. 7 shows spectral performance when PLL control is applied at this point.

Fig. 8(a) and (b) shows the spectrum of a PLL frequency controlled magnetron with a further reduced heater power of $17 \mathrm{~W}$, but plotted on different spans. The $3-\mathrm{dB}$ bandwidth is about $50 \mathrm{kHz}$. The phase here is not locked and this is confirmed by double balanced mixer measurements. With PLL frequency control and when the heater power is reduced to zero the magnetron has a tendency to cut out after several minutes of operation.

Fig. 9 shows return currents from the magnetron anode for three different operating conditions including that of Fig. 8 plotted as a percentages along side the percentage $100-\mathrm{Hz}$ ripple on the $325-\mathrm{V}$ dc input to the power supply chopper.

The mains ripple is an asymmetric triangular wave by virtue of our $50-\mathrm{Hz}$ mains power supply having flattened sinusoidal peaks. The largest waveform in the figure is the anode current ripple when the PLL is not activated and current pulses from the chopper have constant width. This is the full current ripple associated with the $325-\mathrm{V}$ dc input. Were the magnetron to be phase locked as a current controlled oscillator, one would expect a very smooth anode current. When the PLL is activated, the actual anode current still shows a significant residual level of $100-\mathrm{Hz}$ ripple (introduced by the dc input).

The heater is driven by a $50-\mathrm{Hz}$ ac supply and consequently when heater power is applied evidence of $50-\mathrm{Hz}$ ripple appears. The PLL now works to reduce this ripple along with the $100-\mathrm{Hz}$ ripple.

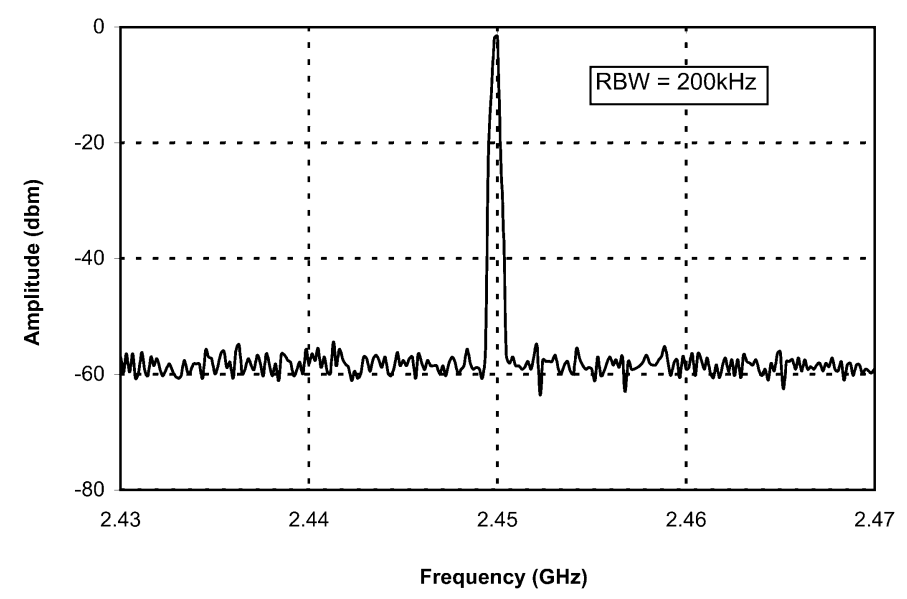

(a)

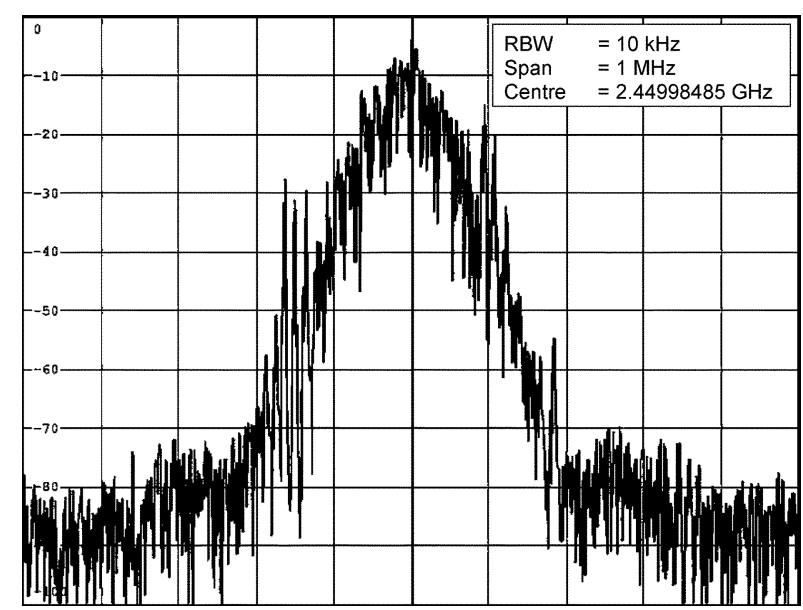

(b)

Fig. 8. (a)Magnetron spectrum with PLL frequency control and with $17 \mathrm{~W}$ heater power. (b) Detail of spectral peak in (a) measured with high specification spectrum analyzer.

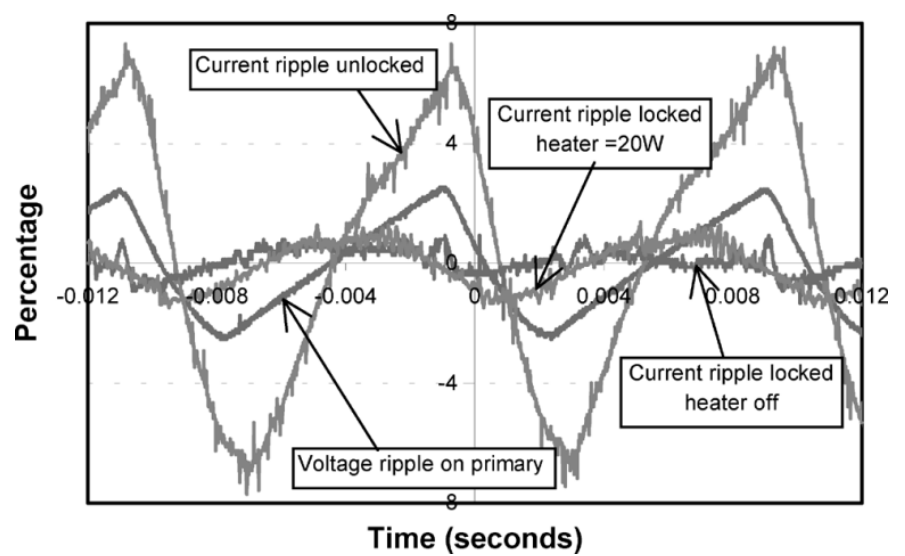

Fig. 9. Anode current ripple against $325-\mathrm{V}$ dc supply voltage ripple for magnetron with and without PLL frequency control and heater power.

The anode current also has a small level of 40-kHz ripple that the control loop is unable to follow. The magnitude of this ripple on the anode current was difficult to measure directly because the power supply was inducing significant voltages on the ground plane. Spectrum analyzer measurements for the locked magnetron indicated that the level of $40-\mathrm{kHz}$ ripple was in the range -35 to $-40 \mathrm{~dB}$ below the dc level. 


\section{MODEl FOR PLL PERFORMANCE}

Defining the phase error at the comparison frequency on the phase frequency detector of the ADF4113 as $\alpha$ then

$$
\alpha=\frac{\omega_{r} t}{N}-\frac{\phi}{S}
$$

where $\omega_{r}$ is the angular frequency of the $10 \mathrm{MHz}$ reference, $N$ is the division ratio for the reference, $\phi$ is the phase of the signal from the magnetron and $S$ is the division ratio for the magnetron signal.

The output of the charge pump is given as

$$
i_{c}=\frac{K_{p}}{2 \pi} \alpha
$$

where $K_{p}=2.5 \mathrm{~mA} \mathrm{rad}^{-1}$ (determined by the ADF4113 ic).

To obtain a simple second-order model for the system it is easiest to neglect the capacitance to ground on the charge pump whose optimum value is typically $5 \%-10 \%$ of the capacitance in the $R C$ filter. This means that the control voltage $V_{c}$ after the loop filter is given as

$$
V_{c}=i_{c} R+\frac{1}{C} \int i_{c} d t+V_{s}
$$

where $R$ is the resistance and $C$ the capacitance of the $R C$ loop filter and $V_{s}$ is an offset voltage needed for start up.

The power supply can be regarded as a current source with a large internal resistance that adds ripple in proportion to the control voltage hence

$$
i=k\{1+X(t)\} V_{c}
$$

where $i$ is the power supply current to the magnetron, $X(t)$ is the fractional ripple and $k$ is determined by the power supply, its control circuitry and its buffer amplifier. The value of $k$ could be adjusted in the range $=0.1-0.4 \mathrm{AV}^{-1}$.

For any small current range the pushing curve of Fig. 1 can be approximated as

$$
\omega=\frac{d \phi}{d t}=\omega_{o}+a i
$$

where $\omega$ is the angular frequency of the magnetron. The coefficients $\omega_{o}$ and $a$ take the values $2 \pi \times 2.44 \mathrm{e} 9$ and $1.57 \mathrm{e} 8$ respectively when $i=0.3 \mathrm{~A}$.

From (2) - (6) one obtains an equation for $\alpha$ as

$$
\begin{aligned}
S \dot{\alpha} & +\left(\omega_{o}-\frac{S}{N} \omega_{r}\right) \\
& +(1+X) \frac{a k K_{p} R}{2 \pi}\left(\alpha+\frac{1}{R C} \int \alpha d t+\frac{2 \pi V_{s}}{R K_{p}}\right)=0
\end{aligned}
$$

If, after a period of time, $\alpha$ tends to a constant value $\alpha_{c}$, taking this period to be the time before $t=0$ and taking $V_{o}$ to be the control voltage at $t=0$, then it follows that for $t>0$

$$
\dot{\alpha}=0 \text { and } \frac{2 \pi V_{s}}{R K_{p}}+\frac{1}{R C} \int \alpha d t=\frac{2 \pi V_{o}}{R K_{p}}+\alpha_{c} t
$$

When $\dot{\alpha}=0$ (2) and (6) give

$$
\omega_{o}-\frac{S}{N} \omega_{r}=-a i
$$

so that $i$ is constant in time.

Substituting (8) and (9) in (7) gives

$$
\frac{i}{1+X}=\frac{k K_{p} R}{2 \pi}\left(\alpha_{c}+\frac{\alpha_{c} t}{R C}+\frac{2 \pi V_{o}}{R K_{p}}\right) .
$$

If $X$ is small (10) can be written

$$
i(1-X)=\frac{k K_{p} R}{2 \pi}\left(\alpha_{c}+\frac{\alpha_{c} t}{R C}+\frac{2 \pi V_{o}}{R K_{p}}\right)
$$

where $X$ is a linear ramp as it would be for a triangular ripple then $X=\dot{X} t$ and the terms increasing linearly with $t$ must balance hence

$$
\alpha_{c}=-\frac{2 \pi C}{k K_{p}} i \dot{X} .
$$

In this equation, the choice ratio $C / k$ is not free and hence cannot be chosen to make the offset error small. For the control loop to work with the 40-kHz switched mode power supply, the current needs to be adjusted at least several times as $\alpha$ swings from zero to a peak value.

The control loop defined by (7) has two natural periods, the under damped period $f_{u}$

$$
f_{u}=\frac{1}{2 \pi} \sqrt{\frac{a k K_{p}}{2 \pi S C}}
$$

and the over damped period $f_{d}$

$$
f_{d}=\frac{1}{2 \pi R C}
$$

both of which need to be less about than $1 / 16$ of the $40 \mathrm{kHz}$ chopper frequency, i.e., less than $2.5 \mathrm{kHz}$. This condition in (13) implies that for $k=0.4 \mathrm{AV}^{-1}$ one must have $C>8$ nF. In practice once $C$ had been chosen $R$ was adjusted to give the best phase jitter performance. Frequency locking was easily achieved for a range of values of $C$ at and above $8 \mathrm{nF}$.

Using the minimum $C$, the phase error $\alpha$ predicted by (12) for a $5 \%$ mains ripple at $100 \mathrm{~Hz}$ becomes $1.56 \times 10^{-4} \mathrm{rad}$. This is the phase error at the PLL phase detector frequency of $200 \mathrm{kHz}$. This translates to a phase error of 1.9 radians at the reference frequency close to $2.45 \mathrm{GHz}$. It is apparent therefore that phase locking cannot be achieved with this level of mains ripple by the method described. Experimentally, the measured phase error for PLL control for our system was never less than $\pi$ radians.

\section{INJECTION LOCKING AND PLL FREQUENCY CONTROL}

By introducing a locking signal into the output waveguide of the magnetron, whose frequency is already controlled by the PLL (phase measured at $200 \mathrm{kHz}$ ), it is possible to get phase locking of the magnetron to that of the reference (phase measured at $2.45 \mathrm{GHz}$ ) with very small power levels whilst subject 


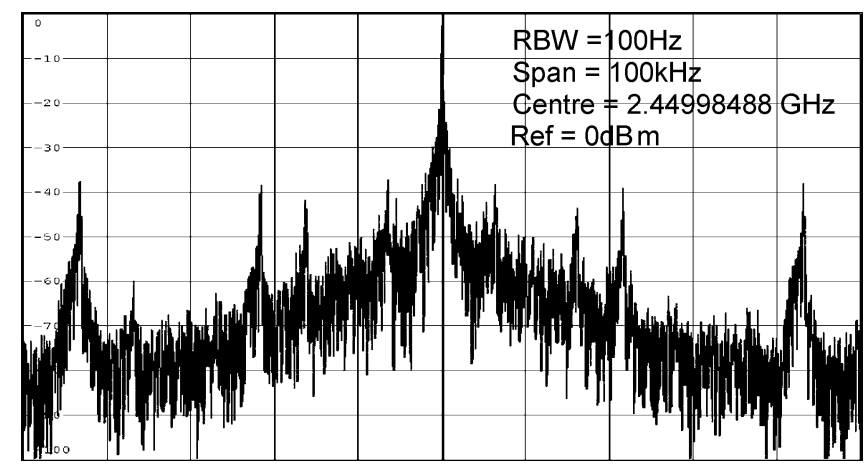

Fig. 10. Magnetron spectrum with 100-mW injection signal, PLL frequency control, and zero heater power.

to approximately $-13 \mathrm{~dB}$ of $100 \mathrm{~Hz}$ and $-35 \mathrm{~dB}$ of $40 \mathrm{kHz}$ power supply ripple.

Injection locking of a magnetron can be understood by the following sequence of events. If a small injection signal, at the same frequency as the magnetron frequency, but at an earlier phase, is introduced into the output cavities of the magnetron, then it adds to the oscillation already there, bringing the overall phase forward by an amount very much less than the phase difference between the signal and magnetron.

The magnetron spokes see the RF field at an earlier relative position and hence, refocus to this earlier position. As the spokes have moved backward so that the negatively charged spokes are closer to the positively charged vanes at the instant of maximum retarding field, the effective capacitance has increased. By the same mechanism for the pushing effect, this increase in capacitance causes the frequency to decrease.

With the magnetron now oscillating at a very slightly lower frequency, the phase of reference can catch up. A similar argument follows for the case when the reference starts in advance of the magnetron phase.

The dynamics of the injection locking process are difficult to model in a simple way. Our experimental results will show that the dc power supply ripple gives a small phase offset, but unlike the offset predicted for frequency locking by varying the anode current, the injection locked offset is proportional to this ripple.

\section{EXPERIMENTAL RESULTS FOR INJECTION LOCKING}

The injection locking signal was derived from the $10-\mathrm{MHz}$ reference by using a second ADF4113 ic to drive a z-comm voltage-controlled oscillator (VCO) V800ME11 at $2.45 \mathrm{GHz}$ as shown in Fig. 2. Careful design is needed to ensure that the signal from the magnetron does not get to the control input of the VCO via the $10 \mathrm{MHz}$ reference. It is essential that the quality of the $2.45-\mathrm{GHz}$ reference signal be monitored while the magnetron is energized to be sure that measurements from the double balanced mixer are meaningful.

Fig. 10 shows the spectrum for the magnetron, injection locked with a signal of $100 \mathrm{~mW}$ over a span of $100 \mathrm{kHz}$.

Spectra plotted for injection levels of between $1 \mathrm{~W}$ and $50 \mathrm{~mW}$ on the same span are almost identical. The $-40 \mathrm{~dB}$ peaks at $+/-21.75 \mathrm{kHz}$ and $43.5 \mathrm{kHz}$ are associated with ripple from the power supply chopper. If the injection level is reduced to $20 \mathrm{~mW}$ the noise floor rises to $-40 \mathrm{dBm}$ across the $100-\mathrm{kHz}$ bandwidth shown in the figures

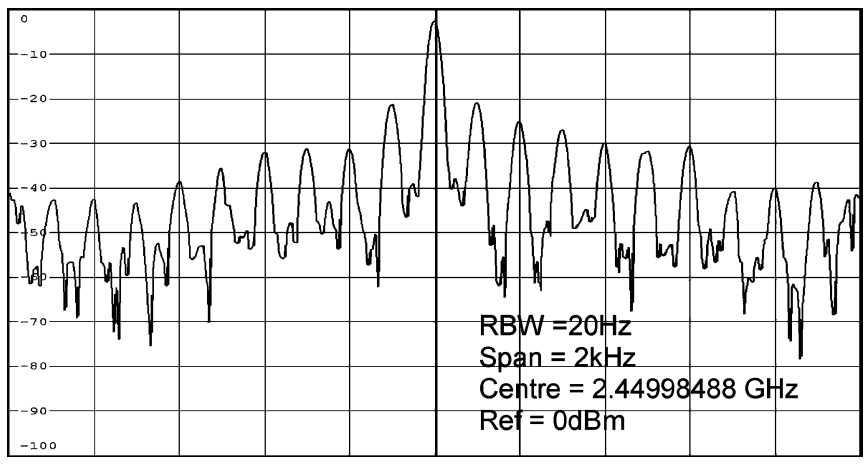

Fig. 11. Magnetron spectrum with 500-mW injection signal, PLL frequency control, and zero heater power.

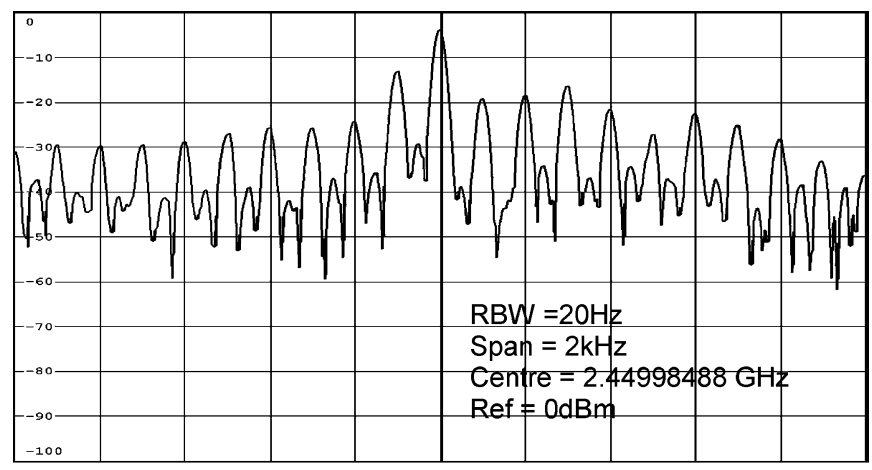

Fig. 12. Magnetron spectrum with 50-mW injection signal, PLL frequency control, and zero heater power.

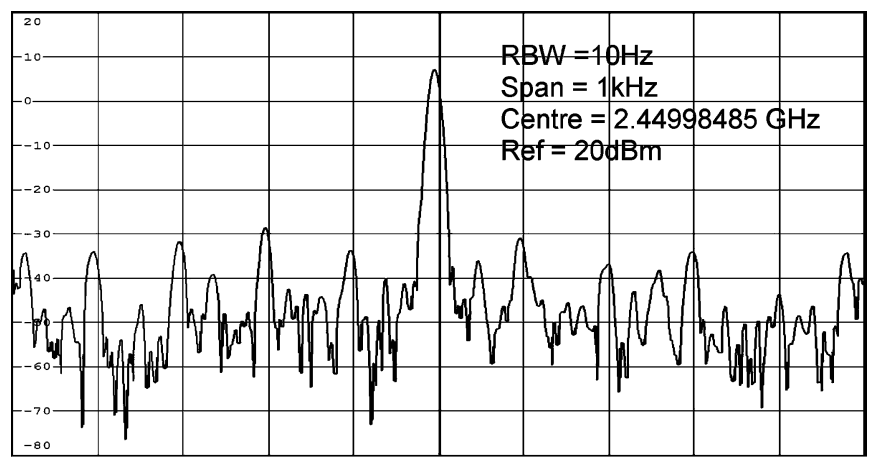

Fig. 13. Injection signal spectrum.

Figs. 11 and 12 show the spectrum for the PLL + injection locked magnetron with injection signal levels of 500, and $50 \mathrm{~mW}$, respectively, and over a span of $2 \mathrm{kHz}$. At the injection level of $50 \mathrm{~mW}$, the $100-\mathrm{Hz}$ side bands are only $10 \mathrm{~dB}$ below the center.

Fig. 13 gives the spectrum for the injection signal measured while the magnetron was energized. The injection signal shows the $100-\mathrm{Hz}$ ripple that exists on the magnetron spectrum but reduced to a level of $-35 \mathrm{dBm}$. The presence of this ripple occurs as a result of pick-up on the VCO input. The pin on the ADF4113 carrying the feedback from the magnetron is physically very close to the pin going to the $10 \mathrm{MHz}$ TCXO oscillator. This pin is also connected to the second ADF4113 that controls VCO providing the injection signal.

Using a Tektronics TDS6804B $8 \mathrm{GHz}$ bandwidth, 20-GHz sampling rate digital storage oscilloscope, it has been possible to record waveforms corresponding to the injection locked 


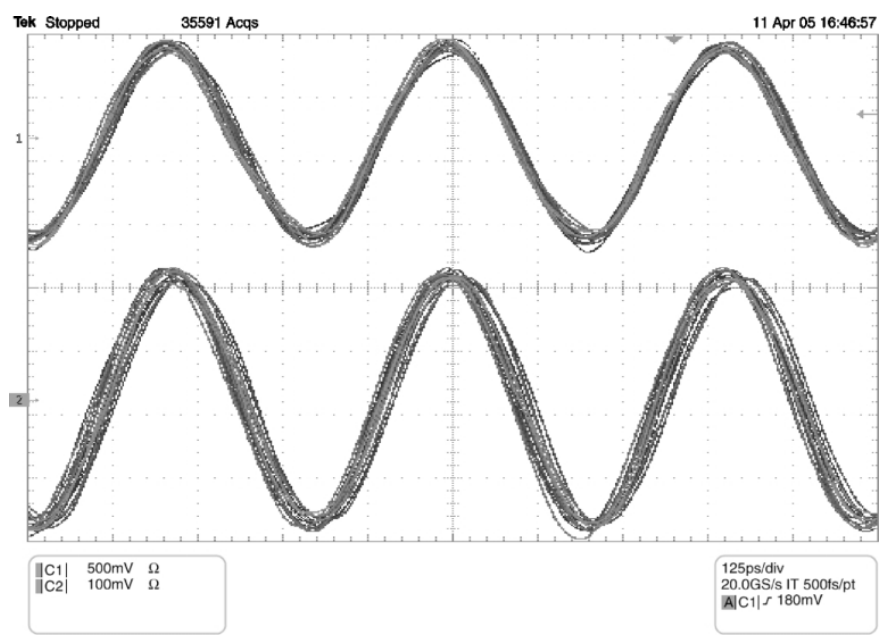

Fig. 14. Magnetron (lower) and reference (upper) waveforms with 500-mW injection signal, PLL frequency control, and zero heater power measured with 50-ms persistence.

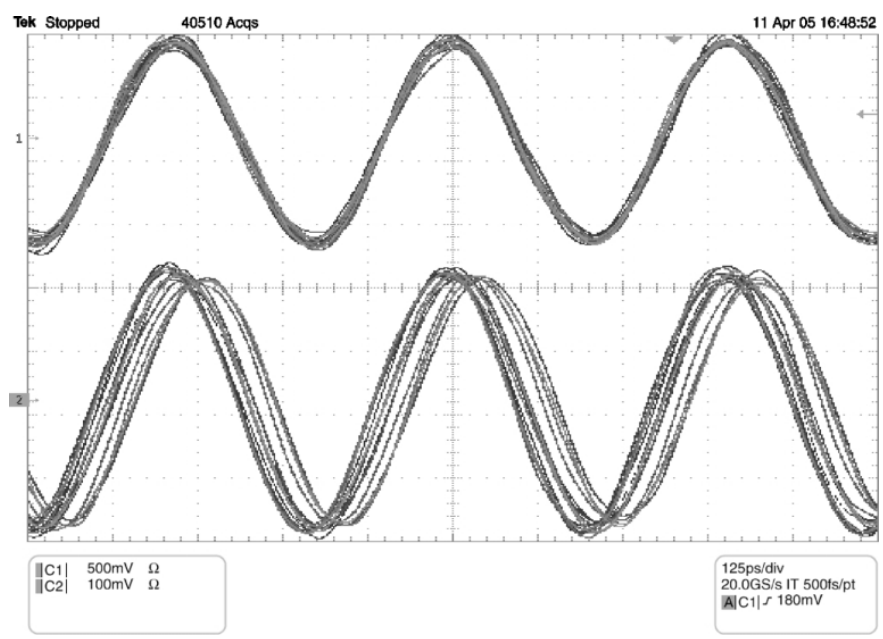

Fig. 15. Magnetron (lower) and reference (upper) waveforms with 100-mW injection signal, PLL frequency control and zero heater power measured with $50 \mathrm{~ms}$ persistence.

magnetron spectra alongside the waveform for the reference. The lower traces in Figs. 14-16 give the magnetron waveforms corresponding to injection levels of 500,100 , and $50 \mathrm{~mW}$, respectively.

The upper waveform in each case is the reference signal. The data has been recorded with a persistence of $50 \mathrm{~ms}$ so that the figures give a sample of waveforms captured over this period. Input noise causes variation in the trigger point and hence the apparent frequency jitter on the injection signal is much larger than one would infer from spectrum of Fig. 13.

Fig. 14 corresponds to Figs. 10 and 11. Fig. 16 corresponds to Fig. 12. The figures illustrate how the phase locking performance deteriorates as the injection level is reduced. The small divisions in Figs. $14-16$ represent a phase angle of $22.5^{\circ}$. It is apparent that measured reference signal starts with a phase variation close $22^{\circ}$. The measured phase variation for the $500-\mathrm{mW}$ injection signal is double this value at about $45^{\circ}$. It is likely therefore that the locking performance at this injection level $\sim+/-10^{\circ}$.

Fig. 17 shows the output from the double balanced mixer for the 100-mW injection level plotted against the ripple on the

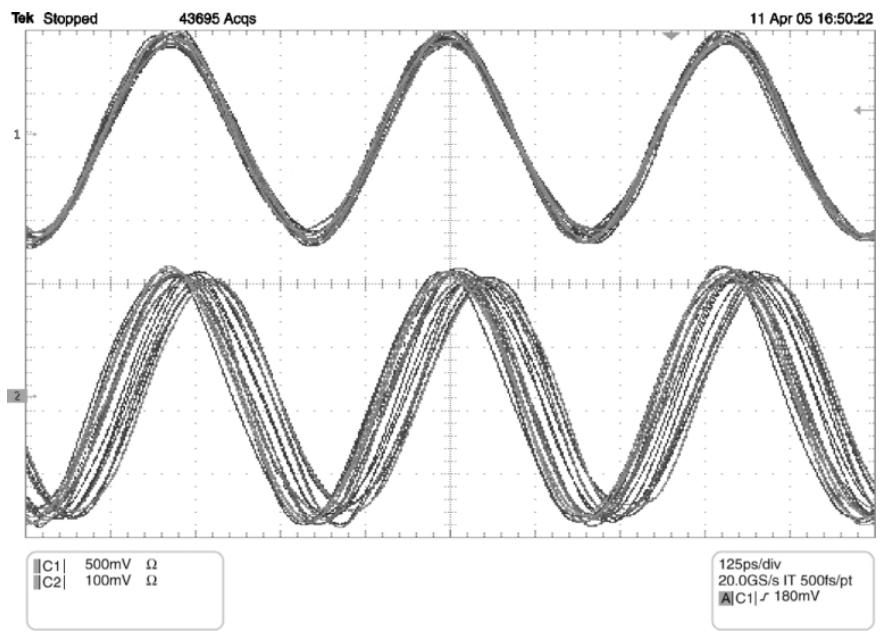

Fig. 16. Magnetron (lower) and reference (upper) waveforms with 50-mW injection signal, PLL frequency control, and zero heater power measured with 50-ms persistence.

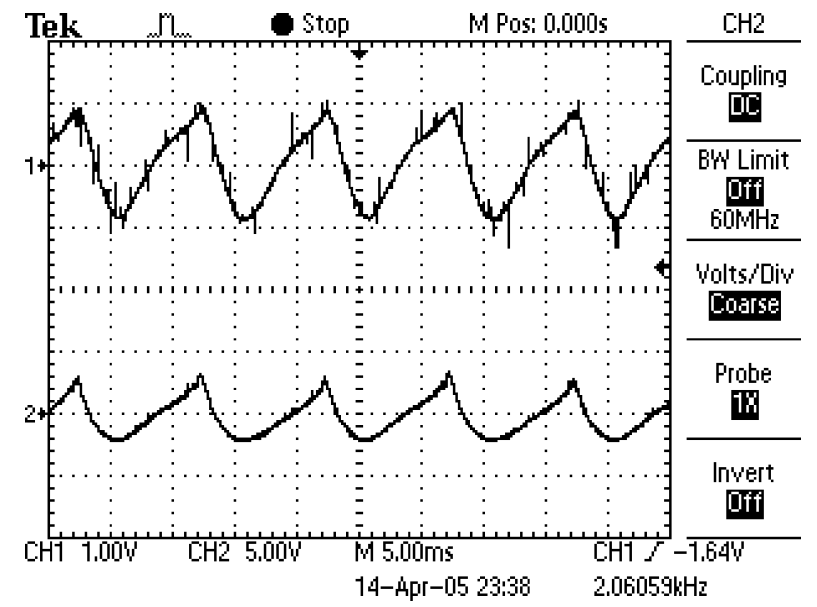

Fig. 17. Double balanced mixer (lower) and anode current ripple (upper) waveforms with $100-\mathrm{mW}$ injection signal, PLL frequency control, and zero heater power.

anode return current from the magnetron. Zero to $\pi$ phase shift corresponds to $13 \mathrm{~V}$ on channel 2 which means that variation of $5 \mathrm{~V}$ in the figure corresponds to $+/-23^{\circ}$ The phase shift follows the anode current ripple. This phase measurement is consistent with the oscilloscope measurement of Fig. 15. Mixer measurements of injection levels of $1 \mathrm{~W}, 500 \mathrm{~mW}$, and $50 \mathrm{~mW}$ gave similar waveforms to Fig. 15 but with phase variations of $+/-9^{\circ}$, $+/-12^{\circ}$ and $+/-44^{\circ}$ respectively.

The anode current shown on channel 1 in Fig. 17 is identical to the anode current of the unlocked system being supplied at constant pulsewidth from the switched mode power supply chopper, see Fig. 9.

It is apparent that the phase error under injection locking follows the mains ripple. This implies that a simple dynamical model describing magnetron injection locking would give a first order control loop. This is exactly the case for Adler's treatment of an injection locked oscillator. He gave the relationship

$$
\sin \Delta \phi=2 Q \frac{E}{E_{\text {inj }}} \frac{\Delta \omega_{o}}{\omega_{o}}
$$


where $\Delta \phi$ is the phase error, $Q$ relates to the oscillator, $\omega_{o}$ is the natural frequency of the oscillator, $\Delta \omega_{0}$ is the difference between the natural frequency and the injection frequency, $E_{\text {inj }}$ is the injected voltage on the grid and $E$ is the input to the grid. For our phase-locked magnetron, the phase error following the mains ripple is consistent with a model of this type where $E$ would relate to the electric field in the magnetron's cavities and $E_{\text {inj }}$ relates to the injected field.

When the magnetron is injection locked the anode current ripple is much larger than when the magnetron just has PLL frequency control. Injection locking makes the phase error much smaller and hence the PLL loop gain becomes too small for the mains ripple to be removed by its action.

\section{CONCLUSION}

This paper paves the way for the development of high-power microwave supplies for industrial processing by combining power from a number of smaller units. Scalable power supplies allow smaller tubes to be mass-produced at low cost. It has been shown that most of the phase error for the injection locked magnetron follows the mains ripple. Where several magnetrons are to be driven from the same mains supply, they would be subject to the same mains ripple and hence the relative phase differences would be much less than the absolute phase differences to the reference.

The investigation here has been constrained to the performance for a single type of power supply. It is of interest to consider now how different levels of ripple and changing load condition affect the locking performance. Recent work by Neculaes et al. [11] has indicated that there is scope for improving the intrinsic noise performance of $\mathrm{CW}$ magnetrons. The techniques suggested may well allow systems to be created with a substantially more robust locking performance at the -40 to $-50 \mathrm{~dB}$ injection level.

\section{ACKNOWLEDGMENT}

The authors wish to thank B. Mackin and A. Verden of Lancaster University for their technical advice and practical assistance. They would also like to thank Tektronics and Rohde \& Schwarz for the loan of equipment.

\section{REFERENCES}

[1] E. Okress, Ed., Crossed-Field Microwave Devices. New York: Academic, 1961, vol. 1, ch. 4.

[2] K. Yamamoto, H. Kuronuma, T. Koinuma, and N. Tashiro, "A study of magnetron noise," IEEE Trans. Electron Devices, vol. ED-34, no. 5, pp. 1223-1226, May 1987.

[3] W. C. Brown, "The high signal to noise ratio of the microwave oven magnetron and evidence of a negative feedback loop to control it," in Proc 1st Int. Workshop Cross-Field Devices, Aug. 1995, pp. 178-187.

[4] R. Adler, "A study of locking phenomena in oscillators," Proc. IRE, pp. 351-357, 1946.

[5] T. Overett, D. B. Remsen, E. Bowles, G. E. Thomas, and R. E. Smith, "Phase locked magnetrons as accelerator sources," in Proc. IEEE Particle Accelerator Conf., 1987, pp. 1464-1465.

[6] S. C. Chen, G. Bekefi, and R. J. Temkin, "Injection locking of a longpulse relativistic magnetron," in Proc. IEEE Particle Accelerator Conf., 1991, pp. 751-753.
[7] N. Shinohara, H. Matsumoto, and K. Hashimoto, "Solar power station/satellite (SPS) with phase controlled magnetrons," IEICW Trans. Electron., vol. E86-C, no. 8, 2003.

[8] J. F. Hull, "Crossed field electron interaction in space charge limited beams," Ph.D. dissertation, Polytechnic Inst. Brooklyn, Brooklyn, NY, 1958.

[9] H. W. Welch, S. Ruthberg, H. W. Batten, and W. Peterson, "Analyis of Dynamic Characteristics of the Magnetron Space Charge Preliminary Results," Univ. Mich., Dept. Elect. Eng., Electron Tube Lab., Ann Arbor, MI, Tech. Rep. 5, 1951.

[10] U. L. Rhode, Microwave and Wireless Synthesizers, Theory \& Design. San Francisco, CA: Jossey-Bass, 1997.

[11] V. B. Neculaes, R. M. Gilgenbach, Y. Y. Lau, M. C. Jones, and W. M. White, "Low-noise microwave oven magnetrons with fast start-oscillation by azimuthally varying axial magnetic fields," IEEE Trans. Plasma Sci., vol. 32, no. 4, pp. 1152-1159, Jul./Aug. 2004.

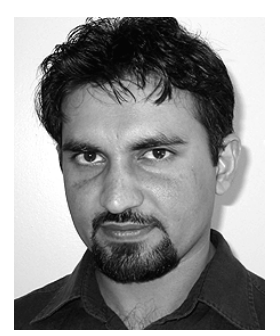

Imran Tahir was born in Burewala, Pakistan, in 1977. He received the B.E. degree from University of Engineering and Technology Lahore, Lahore, Punjab, Pakistan. He is currently pursuing the Ph.D. degree at Lancaster University, Lancaster, U.K., in microwave electronics.

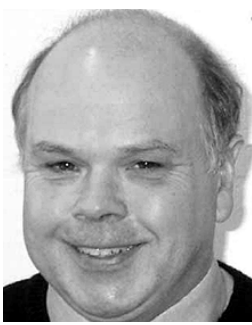

Amos Dexter was born in Carshalton, U.K., in 1956. $\mathrm{He}$ received the B.Sc. degree in mathematics and physics from Bristol University, Bristol, U.K., in 1978 and the Ph.D.degreee in quantum field theory from Liverpool University, Liverpool, U.K., in 1982.

In 1981, he joined the Electricity Council Research Center, Capenhurst, U.K., where he specialized in electromagnetic heating and plasma processing. In 2002, he took up a lectureship with the Engineering Department at Lancaster University, Lancaster, U.K. His research interests now include microwave tube development, RF cavities, RF systems, and multipactors. He is Director of Studies for a joint M.Sc. degree with Strathclyde University, Strathclyde, U.K. on high-power radio frequency science and engineering.

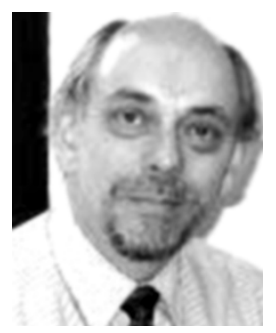

Richard Carter (SM'01) received the degree in physics from the University of Cambridge, Cambridge, U.K., in 1965 and the Ph.D. degree from the University of Wales, Cardiff, U.K., in 1968 for work on the propagation of waves on neutralized ion beams.

From 1968 to 1972 , he worked on high-power traveling-wave tubes as a Development Engineer at English Electric Valve Company, Ltd.. He joined the Engineering Department of the University of Lancaster, Lancaster, U.K., as a Lecturer in 1972 and was promoted to Senior Lecturer in 1986 and Professor of Electronic Engineering in 1996. His research interests include electromagnetics and microwave engineering with particular reference to the theory, design, and computer modeling of microwave tubes and particle accelerators. He is the author of a number of papers, two textbooks on electromagnetics, and a set of video lectures on microwave tubes. He has played key roles in the establishment of the Faraday Partnership in High Power Radio-Frequency Engineering and of the Cockcroft Institute (International Center for Accelerator Science and Technology).

Dr. Carter is a Fellow of the Institution of Electrical Engineers, U.K. 